\section{Indicadores antropométricos na predição de síndrome metabólica em crianças e adolescentes: um estudo de base populacional}

\section{Anthropometric indicators for prediction of metabolic syndrome in children and adolescents: a population-based study}

Rita de Cássia Ribeiro-Silva 1

Thais C. M. Florence 2

Maria Ester Pereira da Conceição-Machado 3

Gilênio Borges Fernandes 4

Ricardo David Couto 5
1-3 Escola de Nutrição. Universidade Federal da Bahia. Av. Araújo Pinho, 32. Canela. Salvador, Bahia, Brasil. CEP: 40.110-150. E-mail: rcrsilva@ufba.br

4 Instituto de Matemática. Universidade Federal da Bahia. Salvador, Bahia, Brasil.

${ }^{5}$ Faculdade de Farmácia. Universidade Federal da Bahia. Salvador, Bahia, Brasil.

\section{Resumo}

Objetivos: avaliar a capacidade dos indicadores antropométricos e pontos de corte na predição da sindrome metabólica (SM) em crianças e adolescentes.

Métodos: estudo transversal, envolvendo amostra probabilística de 879 crianças e adolescentes de ambos os sexos. Dados metabólicos, antropométricos e sociodemográficos foram coletados. Para diagnóstico da SM, foi utilizada a definição modificada do National Cholesterol Education Program's Adult Treatment Panel III (NCEP-ATP III). A capacidade dos indicadores antropométricos na predição do SM foi avaliada por meio da curva Receiver Operating Characteristic (ROC).

Resultados: a prevalência de SM foi de 6,6\%. Na predição da SM, a área sob a curva ROC foi de 0,79 $(0,72 ; 0,85)$ para indice de massa corporal (IMC), de $0,79(0,73 ; 0,85)$ para circunferência da cintura (CC) e de $0,83(0,78 ; 0,89)$ para circunferência da cintura corrigida pela estatura (RCE). O ponto de corte identificado para RCE na predição da SM foi de 0,448 (ambos os gêneros).

Conclusões: todos os indicadores antropométricos utilizados, com pequena superioridade da RCE, foram bons preditores da SM. O ponto de corte identificado para RCE na predição da SM aproxima-se daquele proposto por alguns autores como universal. Sugere-se o uso deste índice dado a sua simples operacionalidade em estudos clínicos e epidemiológicos como preditor da SM.

Palavras-chave Indicadores antropométricos, Síndrome metabólica, Criança, Adolescente 


\section{Introdução}

A obesidade é uma doença crônica, multifatorial, resultante do acúmulo do tecido adiposo, regionalizado ou em todo o corpo, em decorrência da diferença positiva entre o consumo e o gasto energético. ${ }^{1}$ Segundo dados da Pesquisa de Orçamento Familiar (POF) de 2008/2009, cerca de 30\% de crianças na faixa etária entre cinco e nove anos e cerca de $20 \%$ dos adolescentes entre 10 e 18 anos estavam com excesso de peso. O diagnóstico de obesidade foi feito em $4,9 \%$ das crianças e em $14,3 \%$ dos adolescentes. ${ }^{2}$

O excesso de peso (sobrepeso ou obesidade) na infância e adolescência tem sido associado a consequências adversas à saúde em curto e em longo prazo. Estas incluem alteração do metabolismo da glicose (intolerância à glicose ou hiperinsulinemia $\mathrm{e}$ resistência à insulina ou diabetes), dislipidemia (triglicérides altos e HDL-colesterol baixo) e hipertensão arterial, que são fatores de risco para o desenvolvimento de doenças crônicas como o diabetes melito tipo 2 e as doenças cardiovasculares. 1

Diversos indicadores antropométricos têm sido propostos como marcadores preditivos dos distúrbios metabólicos. O índice de massa corporal (IMC) tem sido usado largamente como um indicador de risco para doenças cardiovasculares e diabetes. Contudo, o seu uso tem sido criticado por não se correlacionar com a composição e a distribuição da gordura corporal. Estudo de revisão aponta que os indicadores de obesidade central - a circunferência da cintura (CC), a razão cintura/estatura (RCE) e a razão cintura/quadril (RCQ) - são melhores preditores de alterações metabólicas relacionadas ao risco cardiovascular do que o IMC, principalmente em adultos. ${ }^{3}$

Contudo, o papel da distribuição de gordura na ocorrência de distúrbios metabólicos em crianças e adolescentes ainda permanece inconclusivo. Além disso, em relação a populações latinas ou miscigenadas, ainda não estão disponíveis estudos epidemiológicos apontando a melhor medida antropométrica para esse fim ou seu ponto de corte em crianças e adolescentes. O conhecimento desses pontos de corte é útil na detecção do risco de desenvolvimento de distúrbios metabólicos.

Os distúrbios metabólicos têm surgido cada vez mais precocemente nessa população e são a principal causa de morbimortalidade nos países desenvolvidos e em desenvolvimento. Assim, é premente se desenvolver marcadores de diagnóstico de fácil aplicação, boa precisão e baixo custo na detecção desses eventos, as quais serão de grande utilidade nos serviços de atenção à saúde, além de possibilitar o conhecimento da situação de grupos populacionais específicos diante desses riscos, quando empregadas na pesquisa epidemiológica.

Apesar da relevância do tema, no Brasil, em especial na Bahia, poucos estudos epidemiológicos têm explorado esta temática em crianças e adolescentes.4,5 Nesse sentido, este estudo objetivou avaliar a capacidade dos indicadores antropométricos e pontos de corte na predição da síndrome metabólica (SM) em crianças e adolescentes matriculados na rede pública de ensino da cidade de Salvador-Bahia.

\section{Métodos}

Trata-se de um estudo transversal do qual participaram 879 estudantes de sete a 14 anos, de ambos os sexos. Esses estudantes foram identificados em uma pesquisa mais ampla, que investigou fatores associados à anemia ferropriva em crianças e adolescentes da rede pública de ensino de Salvador (BA). 6 A amostra do estudo foi calculada com base em uma prevalência de anemia de $35 \%$, nível de $95 \%$ de confiança e uma precisão absoluta de $3 \%$. Considerando a possibilidade de perda de $25 \%$, a amostra final foi estimada em 1201 estudantes. O processo de amostragem no referido estudo utilizou a estratificação das escolas em dois níveis (estadual e municipal), seguida pela amostragem por conglomerado em três estágios: o primeiro representado pelos distritos sanitários, o segundo, pelas escolas e o terceiro, pelos alunos.

A amostra foi calculada com base nas informações da matrícula dos escolares da rede pública de Salvador, BA, realizada em 2006, disponibilizadas pela Secretaria de Educação e Cultura do Município e pela Secretaria de Educação do estado da Bahia onde se constatou 217 escolas estaduais (103.924 alunos) e 354 escolas municipais (114.464).

Por questões operacionais elegeram-se seis dos 12 distritos existentes em Salvador, com 117 escolas estaduais, que comportavam 58.059 alunos e 173 municipais, com 56.555. Para alcançar o tamanho amostral foi necessário selecionar em média dez estudantes de cada uma das 58 escolas estaduais e 23 das escolas municipais. Em seguida os estudantes amostrados foram ordenados por série $\left(1^{\mathrm{a}}\right.$ a $\left.8^{\mathrm{a}}\right)$ para posterior amostragem sistemática. Do total de estudantes inicialmente selecionados, registrou-se a perda de $187(15,58 \%)$ deles, devida à recusa, à mudança de endereço ou transferência para outra 
escola.

De um total de 1013 estudantes, 879 aceitaram em participar da coleta de sangue e, portanto foram incluídos nas análises. Registra-se que não foi observada diferença estatisticamente significante entre as características sociodemográficas da amostra original e a utilizada nesse estudo (dados não apresentados em tabela).

Considerando que essa amostra não foi estimada, em face do objetivo investigado neste estudo, decidiu-se por calcular o poder do estudo a posteriori. Assim, essa amostra apresenta poder superior a $90.0 \%$ para avaliar a associação entre a SM e obesidade.

Os dados foram coletados no período de agosto a dezembro de 2010 , por pessoal qualificado e previamente treinado para a coleta. Os gestores das escolas sorteadas receberam uma carta convite para que a escola participasse da pesquisa. Reuniões de esclarecimentos foram realizadas com a comunidade escolar com vistas à adesão ao projeto.

Foram coletados cinco $\mathrm{ml}$ de sangue, após jejum de 12 horas, por via venosa, em ambiente apropriado na escola. As amostras foram devidamente condicionadas e transportadas para o laboratório Central do Complexo Hospitalar Universitário Professor Edgard Santos da Universidade Federal da Bahia. Os níveis séricos de colesterol total, colesterol da lipoproteína de alta densidade (HDL-C) e triglicérides foram determinados por métodos enzimáticos, e o colesterol da lipoproteína de baixa densidade (LDL-C) pela fórmula de Friedewald, quando os triglicérides foram menores que 400 $\mathrm{mg} / \mathrm{dL}$. Os valores de colesterol total $<150 \mathrm{mg} / \mathrm{dL}$, HDL-C $>45 \mathrm{mg} / \mathrm{dL}$ e triglicérides $<100 \mathrm{mg} / \mathrm{dL}$ foram considerados adequados. ${ }^{7}$ A glicemia de jejum foi determinada pelo método enzimático, sendo adequados os valores $<100 \mathrm{mg} / \mathrm{dL} .8$

A pressão arterial das crianças e adolescentes foi aferida em dois momentos, com intervalo de dez minutos aproximadamente. A média entre as medidas representou a medida final utilizada para classificação da pressão arterial. Utilizou-se o esfigmomanômetro aneróide, calibrado no início da coleta dos dados por técnico especializado. A técnica para a aferição das medidas de pressão arterial seguiu as recomendações metodológicas das $V$ Diretrizes Brasileiras de Hipertensão Arterial. ${ }^{9}$ Para a classificação da pressão arterial, foi inicialmente determinado o percentil da estatura/idade de cada estudante, com base na referência do Centers for Disease Control and Prevention dos Estados Unidos.10 De acordo com o percentil de estatura/idade, classificou-se a pressão arterial conforme o sexo, utilizando-se a recomendação do The Fourth Report on the Diagnosis, Evaluation, and Treatment of High Blood Pressure in Children and Adolescents. 11

As medidas antropométricas foram coletadas na escola, de maneira padronizada, seguindo os procedimentos preconizados pelo Anthropometric Standartization Reference Manual.12 O peso foi obtido com o auxílio de balança microeletrônica, marca Marte, modelo PP 200-50, com capacidade para $199,95 \mathrm{~kg}$ e precisão de 50 gramas. Para a obtenção da estatura, foi utilizado um estadiômetro da marca Leicester Hight Measure, graduado em décimos de centímetros. O peso corpóreo e a altura foram tomados em duplicata por dois antropometristas independentes, que registraram os resultados em formulário próprio, admitindo-se variação mínima de $0,5 \mathrm{~cm}$ para medida de estatura e $100 \mathrm{~g}$ para medida de peso.

$\mathrm{O}$ índice de massa corporal [IMC $=$ peso $(\mathrm{kg}) /$ estatura $(\mathrm{m})^{2}$ ] foi utilizado para o diagnóstico do estado nutricional, avaliado pela antropométrica, adotando-se os percentis segundo idade e sexo, propostos pela Organização Mundial da Saúde,13 categorizado em: magreza ( $<$ percentil 3 ), adequado ( $\geq$ percentil $3 \mathrm{e} \leq$ percentil 85 ), sobrepeso ( $>$ percentil 85 e $\leq$ percentil 97) e obesidade (percentil $>97$ ). Para análise, foram agregadas as categorias sobrepeso e obesidade. Portanto, os indivíduos com excesso de peso conferiam IMC situados no percentil igual ou acima de 85 .

A medida da circunferência da cintura (CC) foi realizada no ponto médio entre a crista ilíaca e a face externa da última costela, por meio de fita inelástica, graduada em centímetros e milímetros, evitando compressão da pele. $\mathrm{O}$ indivíduo posicionou-se com os pés juntos, braços relaxados e soltos ao longo do corpo com o peso apoiado em ambos os pés e a leitura foi realizada no milímetro mais próximo, ao final da expiração, com o indivíduo respirando suavemente. As medidas foram ser realizadas em duplicata, aceitando-se variações de $0,5 \mathrm{~cm}$ entre as duas medidas, adotando-se a média entre os valores como a medida final. A classificação para definição de obesidade abdominal foi feita de acordo com os pontos de corte por idade e sexo propostos por Taylor et al., 14 que considera elevado valor acima do percentil 80 . A relação $\mathrm{CC}(\mathrm{cm}) /$ Estatura $(\mathrm{cm})(\mathrm{RCE})$ foi obtida pelo quociente da $\mathrm{CC}$ e estatura, adotandose como ponto de corte para definição de obesidade abdominal o valor $\geq 0,5.15$

Para diagnóstico da Síndrome Metabólica, foi utilizada a definição modificada do National Cholesterol Education Program's Adult Treatment 
Panel III (NCEP-ATP III), 16 que consiste na presença de pelo menos três dos seguintes fatores: obesidade (caracterizada pela obesidade abdominal$\mathrm{CC}$ ), dislipidemia (altos níveis de triglicerídeos ou baixo nível de HDL), hipertensão arterial e hiperglicemia de jejum. Entretanto, os pontos de cortes foram adaptados à idade da população estudada. A variável foi analisada de forma dicotômica, a saber: indivíduos não portadores da síndrome metabólica (categoria de referência - 0) e indivíduos portadores da síndrome metabólica (categoria de exposição - 1).

A partir de um questionário estruturado foram obtidos dados de escolaridade materna (indicador proxy das condições socioeconômicas da família). A escolaridade materna foi categorizada em dois níveis, conforme a última série escolar cursada: $\mathrm{I} \leq$ 4a série, II $\geq 5$ a série ${ }_{(\text {categoria de referência). }}$. As variáveis demográficas foram: sexo (masculino (categoria de referência) , feminino) e faixa etária $\left(<10 \operatorname{anos}_{\text {(categoria de }}\right.$ referência) $\mathrm{e} \geq 10$ anos).

Para o processamento e construção do banco de dados, foi utilizado o Epi-Info versão 6.04 (Centros de Controle e Prevenção de Doenças, em Atlanta, GA, USA). Adotou-se a digitação dupla dos dados, após os questionários serem revisados e os erros decorrentes da codificação realizada inicialmente em campo corrigidos. A verificação das frequências simples das variáveis e o exame da coerência entre perguntas e respostas foram os meios utilizados para a limpeza do banco de dados.

Para a comparação das prevalências de SM segundo as características da população estudada empregou-se o teste qui-quadrado $\left(\chi^{2}\right)$ de Pearson.

Análises de Curvas Receiver Operating Characteristic (ROC) foram utilizadas para avaliar e comparar a capacidade de identificar a SM pelos índices antropométricos IMC, RCE e CC. As áreas sob a curva ROC fornecem a probabilidade global de esses índices classificarem corretamente a presença ou a ausência de SM. Um teste perfeito tem uma área sob a curva ROC igual a 1,0, já uma área igual a 0,5 significa que a performance do teste não é melhor do que ao acaso. O IC95\% é outro determinante da capacidade preditiva. Portanto, para o indicador antropométrico verificado ser considerado preditor significativo da SM, o limite inferior do IC (Li-IC) não pode ser $<0,50.17$ Foram também identificados os pontos de corte da RCE, com melhor capacidade preditiva de SM, local onde as curvas de sensibilidade e especificidade se cruzam; valores com sensibilidade e especificidade mais próximos entre si.

Nas análises estatísticas adotaram-se os testes bicaudais e um nível de significância de 5\%. Para as análises utilizou-se o software Statistical Package for the Social Sciences (SPSS), v. 17.0.

Os pais ou responsáveis pelos estudantes que concordaram com a participação deles na pesquisa assinaram o Termo de Consentimento Livre e Esclarecido; os analfabetos o fizeram por meio da impressão digital. O estudo teve seu protocolo aprovado pelo Comitê de Ética em Pesquisa (CEP) do Instituto de Saúde Coletiva (ISC) da Universidade Federal da Bahia (no 043-05/CEPISC).

\section{Resultados}

Participaram do presente estudo 879 estudantes, sendo $73,6 \%$ maiores de 10 anos e $50,7 \%$ do sexo masculino. A prevalência de SM foi de 6,6\% (IC95\% $0,04 ; 0,08)$. Verificou-se associação da SM com sexo feminino $(p=0,02)$, HDLc alterado $(p<0,001)$, triglicérides alterado $(p<0,001)$, níveis pressóricos alterados $(p<0,001)$, obesidade $(p<0,001)$, adiposidade abdominal, avaliado pela CC $(p<0,001)$, e adiposidade abdominal, avaliado pela RCE $(p<0,001)$. Não se verificou associações estatisticamente significantes da SM e demais variáveis (Tabela 1)

$\mathrm{Na}$ predição da SM, a área sob a curva ROC foi de $0,79(0,72 ; 0,85)$ para IMC, de $0,79(0,73 ; 0,85)$ para $\mathrm{CC}$ e de $0,83(0,78 ; 0,89)$ para RCE. Nas Figuras 1, 2 e 3 são apresentados os pontos de corte para RCE com maior capacidade preditiva de SM. Esses pontos foram de 0,448 para ambos os sexos, sendo 0,447 para os participantes do sexo feminino e 0,445 para os do sexo masculino. Nesses pontos de corte, os valores de sensibilidade e especificidade foram semelhantes, pois correspondem ao local no qual as curvas de sensibilidade e especificidade se cruzam $(77,0 \%$ para ambos os sexos, $78 \%$ e $76 \%$, respectivamente, para o sexo masculino e feminino). 
Prevalência da síndrome metabólica segundo caracterização dos estudantes matriculados na rede pública de ensino da cidade de Salvador, BA. 2009.

\begin{tabular}{|c|c|c|c|c|c|}
\hline \multirow{2}{*}{ Características } & \multicolumn{2}{|c|}{ População } & \multicolumn{2}{|c|}{ Síndrome metabólica } & \multirow[b]{2}{*}{$p$} \\
\hline & $\mathrm{n}$ & $\%$ & $\mathrm{n}$ & $\%$ & \\
\hline Sexo & & & & & 0,02 \\
\hline Masculino & 446 & 50,7 & 21 & 4,7 & \\
\hline Feminino & 433 & 49,3 & 37 & 8,5 & \\
\hline Idade (anos) & & & & & 0,92 \\
\hline$<10$ & 232 & 26,4 & 15 & 6,5 & \\
\hline$\geq 10$ & 647 & 73,6 & 43 & 6,5 & \\
\hline Escolaridade materna* & & & & & 0,27 \\
\hline 5 série & 272 & 31,6 & 42 & 7,1 & \\
\hline$\geq 5$ série & 590 & 68,4 & 14 & 5,1 & \\
\hline Níveis pressóricos & & & & & $<0,001$ \\
\hline < percentil 95 - normal & 771 & 87,7 & 23 & 3,0 & \\
\hline$\geq$ percentil 95 - alterado & 108 & 12,3 & 35 & 32,4 & \\
\hline HDLC & & & & & $<0,001$ \\
\hline$\geq 45 \mathrm{mg} / \mathrm{dL}$ - desejável & 600 & 68,3 & 12 & 2,0 & \\
\hline$<45 \mathrm{mg} / \mathrm{dL}$ - alterado & 279 & 31,7 & 46 & 16,5 & \\
\hline Triglicérides & & & & & $<0,001 \#$ \\
\hline$<100$ mg/dL - normal & 615 & 70,0 & 3 & 0,5 & \\
\hline$\geq 100 \mathrm{mg} / \mathrm{dL}$ - elevado & 264 & 30,0 & 55 & 20,8 & \\
\hline Glicemia* & & & & & $0,48 \#$ \\
\hline$<100$ mg/dL - normal & 864 & 98,5 & 58 & 6,7 & \\
\hline$\geq 100 \mathrm{mg} / \mathrm{dL}$ - elevado & 13 & 1,5 & 0 & 0,0 & \\
\hline Estado nutricional (IMC) & & & & & $<0,001$ \\
\hline Sem excesso de peso & 770 & 87,6 & 25 & 3,2 & \\
\hline Com excesso de peso & 109 & 12,4 & 33 & 30,3 & \\
\hline Circunferência da cintura & & & & & $<0,001$ \\
\hline Sem obesidade abdominal & 717 & 81,6 & 13 & 1,8 & \\
\hline Com obesidade abdominal & 162 & 18,4 & 45 & 27,8 & \\
\hline Relação Cintura/Estatura & & & & & $<0,001$ \\
\hline Sem obesidade abdominal & 830 & 94,4 & 41 & 4,9 & \\
\hline Com obesidade abdominal & 49 & 5,6 & 71 & 34,7 & \\
\hline
\end{tabular}

*Dados faltantes; IMC= Índice de Massa Corporal; HDL-c= colesterol contido na lipoproteína de alta densidade; \#Teste exato de Fischer. 
Relação cintura/estatura e síndrome metabólica: sensibilidade, especificidade e melhor ponto de corte em crianças e adolescentes, de ambos os sexos, matriculadas na rede pública de ensino da cidade de Salvador, BA. 2009.

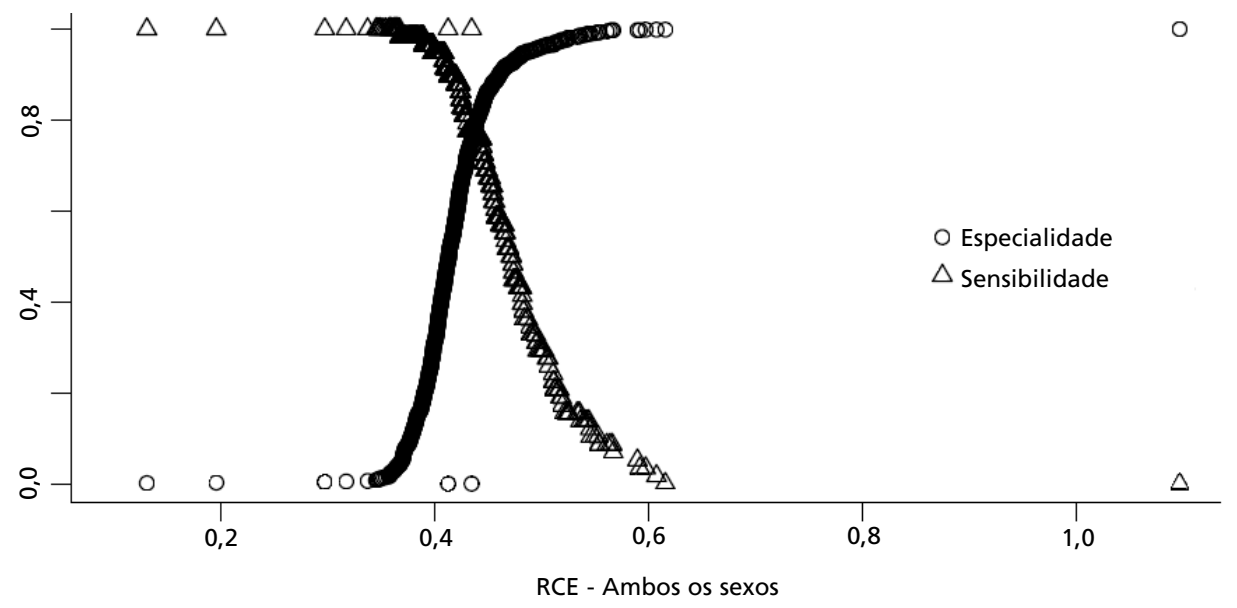

Figura 2

Relação cintura/estatura e síndrome metabólica: sensibilidade, especificidade e melhor ponto de corte em crianças e adolescentes, do sexo feminino, matriculadas na rede pública de ensino da cidade de Salvador, BA. 2009.

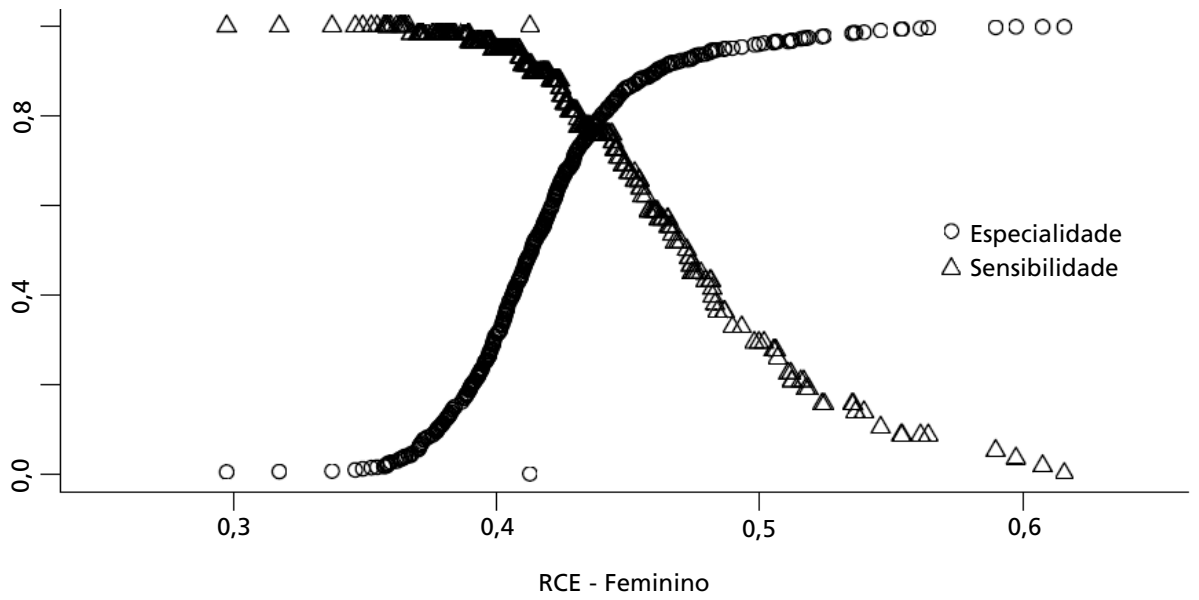


Relação cintura/estatura e síndrome metabólica: sensibilidade, especificidade e melhor ponto de corte em crianças e adolescentes, do sexo masculino, matriculadas na rede pública de ensino da cidade de Salvador, BA. 2009.

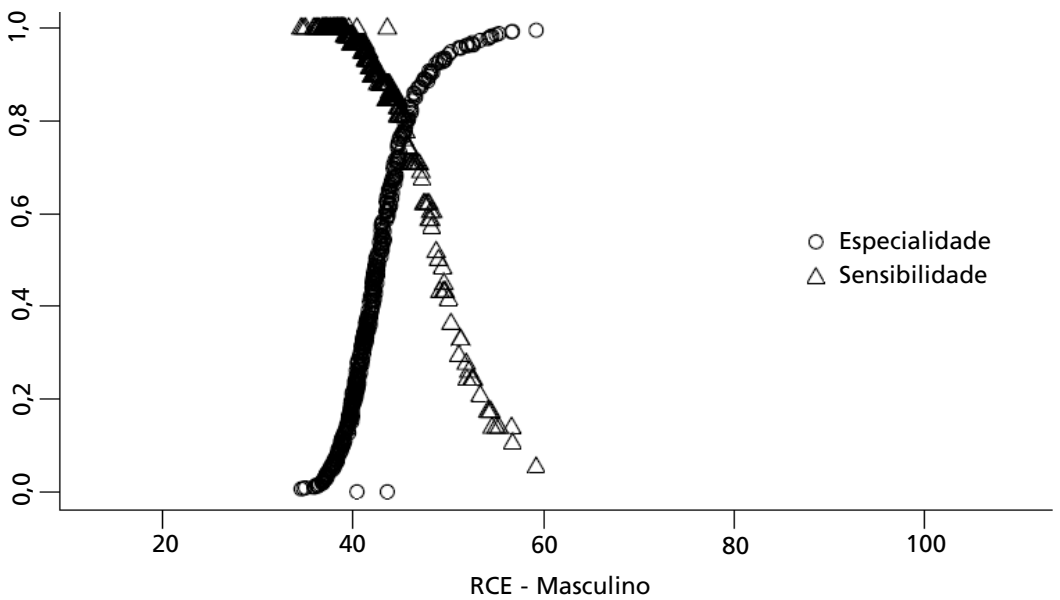

\section{Discussão}

Este estudo examinou a capacidade dos indicadores antropométricos (IMC, CC e RCE), além dos pontos de corte com maior capacidade preditiva da RCE, na identificação da SM em uma amostra representativa de crianças e adolescentes em Salvador, Bahia. Trata-se de uma investigação desenvolvida em um cenário epidemiológico caracterizado por frequência elevada de dislipidemia, excesso de peso e hipertensão arterial. A prevalência de SM no grupo estudado foi de $6,6 \%$, sendo mais elevada entre os adolescentes do sexo feminino, que apresentaram perfil lipídico alterado (hipertrigliceridemia e baixo nível sérico de HDL-C), níveis pressóricos elevados e excesso de peso.

A prevalência de SM encontrada aproxima-se daquela observada em estudos que utilizaram os mesmos critérios diagnósticos para identificação da síndrome. ${ }^{18,19}$ A heterogeneidade de definições e de pontos de corte para os componentes da SM pode explicar, ao menos em parte, as diferentes prevalências relatadas na literatura; ainda assim, admite-se que estas vêm aumentando tanto em países desenvolvidos quanto naqueles em desenvolvimento. No Brasil, a prevalência varia entre $0 \%$ e $42 \%$, sendo mais acentuada nos indivíduos com excesso de peso; esse é um indicativo de que a ocorrência da SM tem, no excesso de gordura corporal, seu mais importante fator de risco. 20

Muitas investigações têm buscado identificar o poder preditivo dos indicadores antropométricos para as doenças cardiometabólicas. ${ }^{3} \mathrm{~A}$ análise da sensibilidade e especificidade por meio da construção de curvas ROC tem sido amplamente usada em estudos epidemiológicos para a determinação de pontos de corte de indicadores antropométricos na predição de SM. Esse tipo de análise não só permite a identificação do melhor ponto de corte como também fornece a área sob a curva que traduz o poder de discriminação de um indicador para um determinado desfecho.

No presente estudo, foi possível observar similar performance da circunferência da cintura [CC: 0,79 $(0,73-0,85)]$ e do índice de massa corporal [IMC= $0,79(0,72-0,85)]$ na predição da SM. Por outro lado, a razão cintura/estatura (RCE) revelou-se levemente melhor preditora da $\mathrm{SM}$, com valor da área sob a curva ROC para esse desfecho de $0,83[\mathrm{RCE}=0,83$ $(0,78-0,89)]$. Esses resultados se sustentam na plausibilidade biológica e corroboram a tese de que a gordura corporal, especialmente a de localização abdominal, está relacionada à SM, como apoiado em outros estudos, que evidenciaram a superioridade da mensuração da gordura abdominal, especialmente quando se utiliza a RCE, na detecção de risco cardiovascular e distúrbios metabólicos em crianças e adolescentes, ${ }^{21}$ sendo um bom preditor na discriminação da SM. ${ }^{3}$

Múltiplos mecanismos biológicos têm sido implicados na mediação dos efeitos adversos do excesso de adiposidade sobre a saúde, entretanto, os caminhos exatos são desconhecidos. Além dos mecanismos que envolvem secreção de adipocinas e outras substâncias vasoativas, a gordura visceral 
parece ser mais sensível à lipólise, comparada à gordura subcutânea, o que pode contribuir para o seu papel na manifestação dos fatores de risco da SM. ${ }^{22}$

$\mathrm{O}$ acúmulo de gordura intra-abdominal, que também está associado com maiores diâmetros da aorta abdominal, é um fenômeno que tem sido relacionado a distúrbios metabólicos (níveis pressóricos elevados e dislipidemia). ${ }^{23}$ Registra-se, contudo, que o aumento excessivo de gordura corporal independentemente do seu depósito, pode causar prejuízo ao metabolismo de carboidratos e lipídios, bem como produção exacerbada de fatores potencializadores da SM. ${ }^{24}$

Foi identificado o ponto de corte da RCE para ambos os sexos de 0,448, que assemelha-se àquele proposto por McCarthy et al., 25 como universal no rastreamento do risco cardiovascular, tanto para crianças e adolescentes quanto para adultos. Citamse estudos que têm observado pontos de corte da RCE na detecção do distúrbio metabólico associado ao excesso de gordura abdominal de 0,47 para crianças brasileiras de 6 a 10 anos de idade; 26 de 0,41 para adolescentes africanos de 9 a 15 anos de idade27 e de 0,48 e 0,47 para adolescentes australianos, meninos e meninas respectivamente, de 8 a 16 anos de idade. 28

São mencionados ainda os autores que propuseram pontos de corte da RCE a partir daqueles do IMC, utilizados para diagnóstico de sobrepeso e obesidade em crianças e adolescentes. Kahn et al. 29 propuseram, para indivíduos de 4 a 17 anos, os pontos de corte de RCE de 0,490 para o risco de sobrepeso e 0,539 para sobrepeso, equivalentes, respectivamente, aos percentis 85 e 95 do IMC das curvas do Centers for Disease Control (CDC). Já Weili et al.,30 para indivíduos de 8 a 18 anos, propuseram pontos de corte da RCE com diferenças entre os sexos: de 0,44 para o sobrepeso nos dois sexos, e de 0,485 em meninos e 0,475 em meninas para a obesidade, equivalentes, respectivamente, ao IMC de $24 \mathrm{~kg} / \mathrm{m}^{2}$ e $28 \mathrm{~kg} / \mathrm{m}^{2}$.

Ressalta-se que os pontos de corte da RCE propostos no presente estudo, de 0,445 para os meninos e de 0,447 para as meninas, se adequam ambos à seguinte mensagem dirigida a crianças, adolescentes e adultos: é saudável manter sua circunferência da cintura abaixo da metade de sua estatura. 25

É importante salientar que a principal limitação desta investigação está no fato de se tratar de um estudo transversal. Por conseguinte, o desenho adotado permite apenas observar associações entre os eventos estudados, não sendo possível demonstrar uma relação de causa e efeito entre tais eventos. Estudos longitudinais futuros para avaliar os indicadores antropométricos na predição de doença cardiovascular e mortalidade são necessários para elucidar completamente as relações relatadas. Em adição, cita-se o limite imposto pela idade das crianças estudadas, sobre a qual não há consenso em relação à definição de SM.

Em síntese, todos os indicadores antropométricos utilizados, com pequena superioridade da RCE, se apresentaram como bons preditores da SM. O ponto de corte da RCE com maior capacidade preditiva de SM, para ambos o sexos, foi de 0,448 . Sugere-se, com base nos resultados do presente estudo, que a RCE é o índice que aqui se recomenda em razão da sua simples operacionalidade em estudos clínicos e epidemiológicos como preditor da SM. Cabe ainda ressaltar a necessidade da realização de programas de controle do excesso de peso em crianças e adolescentes, visto que os obesos têm maior probabilidade de desenvolver a síndrome metabólica.

\section{Referências}

1. WHO (World Health Organization). Obesity: preventing and managing the global epidemic. Technical Report Series. Geneva; 2000. Disponível em: http://smsaint.org/ media/1286/Obesity-Preventing-and-Managing-Globalepidemic.pdf

2. IBGE (Instituto Brasileiro de Geografia e Estatística). Antropometria e estado nutricional de crianças, adolescentes e adultos no Brasil. Pesquisa de Orçamento Familiar (POF) 2008-2009. Rio de Janeiro; 2010.

3. Ashwell M, Gunn P, Gibson S. Waist-to-height ratio is a better screening tool than waist circumference and BMI for adult cardiometabolic risk factors: systematic review and meta-analysis. Obes Rev. 2012; 13 (3): 275-86
4. Ferreira AP, Oliveira CE, Franca NM. Metabolic syndrome and risk factors for cardiovascular disease in obese children: the relationship with insulin resistance (HOMA-IR). J Pediatr (Rio J). 2007; 83 (1): 21-6.

5. Ferreira AP, Ferreira CB, Brito CJ, Pitanga FJG, Moraes CF, Naves LA, Nóbrega OT, França NM. Predição da síndrome metabólica em crianças por indicadores antropométricos. Arq Bras Cardiol. 2011; 96: 121-5.

6. Borges CQ, Silva RdCR, Assis AMO, Pinto EdJ, Fiaccone RL, Pinheiro SMC. Fatores associados à anemia em crianças e adolescentes de escolas públicas de Salvador, Bahia, Brasil. Cad Saúde Pública. 2009; 25: 877-88. 
7. Back Giuliano Ide C, Caramelli B, Pellanda L, Duncan B, Mattos S, Fonseca FH. [I guidelines of prevention of atherosclerosis in childhood and adolescence]. Arq Bras Cardiol. 2005; 85 (Suppl 6): 4-36

8. Basevi V, Di Mario S, Morciano C, Nonino F, Magrini N. Comment on: American Diabetes Association. Standards of medical care in diabetes--2011. Diabetes Care 2011; 34 (Suppl. 1): S11-S61.

9. Mion Jr D, Kohlmann Jr O, Machado CA, Amodeo C, Gomes MAM, Praxedes JN, Nobre F, Brandão A, Zanella MT, Gusmão JL. V Brazilian Guidelines in Arterial Hypertension. Arq Bras Cardiol. 2007; 89 (3): e24-79.

10. Centers for Disease Control and Prevention/National Center for Health Statistics. CDC growth charts: United States, 2000. [acesso em 20 Jan 2014]. Disponível em: http://www.cdc.gov/growthcharts

11. National High Blood Pressure Education Program Working Group on High Blood Pressure in Children and Adolescents. The fourth report on the diagnosis, evaluation, and treatment of high blood pressure in children and adolescents. Pediatrics. 2004; 114 (Suppl. 2 4th Report): 555-76.

12. Lohman TG, Roche AF, Martorell R. Anthropometric standardization reference manual. Champaign, IL: Human Kinetics Books; 1988. vi, 177 p.

13. Onis M, Onyango AW, Borghi E, Siyam A, Nishida C, Siekmann J. Development of a WHO growth reference for school-aged children and adolescents. Bull World Health Organ. 2007; 85 (9): 660-7

14. Taylor RW, Jones IE, Williams SM, Goulding A. Evaluation of waist circumference, waist-to-hip ratio, and the conicity index as screening tools for high trunk fat mass, as measured by dual-energy X-ray absorptiometry, in children aged 3-19 y. Am J Clin Nutr. 2000; 72 (2): 490-5.

15. Li C, Ford ES, Mokdad AH, Cook S. Recent trends in waist circumference and waist-height ratio among US children and adolescents. Pediatrics. 2006; 118 (5): e1390-8.

16. Executive Summary of The Third Report of The National Cholesterol Education Program (NCEP) Expert Panel on Detection, Evaluation, And Treatment of High Blood Cholesterol In Adults (Adult Treatment Panel III). JAMA. 2001; 285 (19): 2486-97.

17. Erdreich LS, Lee ET. Use of relative operating characteristic analysis in epidemiology. A method for dealing with subjective judgement. Am J Epidemiol. 1981; 114 (5): 64962 .

18. Cook S, Weitzman M, Auinger P, Nguyen M, Dietz WH. Prevalence of a metabolic syndrome phenotype in adolescents: findings from the third National Health and Nutrition Examination Survey, 1988-1994. Arch Pediatr Adolesc Med. 2003; 157 (8): 821-7.

Recebido em 30 de outubro de 2013

Versão final apresentada em 26 de março de 2014

Aprovado em 7 de abril de 2014
19. Duncan GE, Li SM, Zhou XH. Prevalence and trends of a metabolic syndrome phenotype among U.S. Adolescents, 1999-2000. Diabetes Care. 2004; 27 (10): 2438-43.

20. Moraes ACFd, Fulaz CS, Netto-Oliveira ER, Reichert FF. Prevalência de síndrome metabólica em adolescentes: uma revisão sistemática. Cad Saúde Pública. 2009; 25: 1195202.

21. Freedman DS, Kahn HS, Mei Z, Grummer-Strawn LM, Dietz WH, Srinivasan SR, Berenson GS. Relation of body mass index and waist-to-height ratio to cardiovascular disease risk factors in children and adolescents: the Bogalusa Heart Study. Am J Clin Nutr. 2007; 86 (1): 33-40.

22. Ribeiro Filho FF, Mariosa LS, Ferreira SRG, Zanella MT. Gordura visceral e síndrome metabólica: mais que uma simples associação. Arq Bras Endocrinol Metabol. 2006; 50: $230-8$.

23. Gorter PM, Visseren FL, Moll FL, van der Graaf Y. Intraabdominal fat and metabolic syndrome are associated with larger infrarenal aortic diameters in patients with clinically evident arterial disease. J Vasc Surg. 2008; 48 (1): 114-20.

24. Hermsdorff HHM, Monteiro JBR. Gordura visceral, subcutânea ou intramuscular: onde está o problema? Arq Bras Endocrinol Metabol. 2004; 48: 803-11.

25. McCarthy HD, Ashwell M. A study of central fatness using waist-to-height ratios in UK children and adolescents over two decades supports the simple message--'keep your waist circumference to less than half your height'. Int J Obes (Lond). 2006; 30 (6): 988-92.

26. Kuba VM, Leone C, Damiani D. Is waist-to-height ratio a useful indicator of cardio-metabolic risk in 6-10-year-old children? BMC Pediatr. 2013; 13: 91.

27. Motswagole BS, Kruger HS, Faber M, van Rooyen JM, Ridder JH. The sensitivity of waist-to-height ratio in identifying children with high blood pressure. Cardiovasc J Afr. 2011; 22 (4): 208-11

28. Nambiar S, Hughes I, Davies PS. Developing waist-toheight ratio cut-offs to define overweight and obesity in children and adolescents. Public Health Nutr. 2010; 13 (10): 1566-74.

29. Kahn HS, Imperatore G, Cheng YJ. A population-based comparison of BMI percentiles and waist-to-height ratio for identifying cardiovascular risk in youth. J Pediatr. 2005; 146 (4): $482-8$.

30. Weili Y, He B, Yao H, Dai J, Cui J, Ge D, Zheng Y, Li L, Guo Y, Xiao K, Fu X, Ma D. Waist-to-height ratio is an accurate and easier index for evaluating obesity in children and adolescents. Obesity (Silver Spring). 2007; 15 (3): 748- 